\title{
Los conceptos de
}

\section{Hombre y Cultura}

en la época de la

\section{Técnica}

ME ADOLFOLEONGRISALES VARGAS

Profesor Universidad de Caldas

Departamento de Filosofin

Departamento Ciencias Humanas

Universidad Autónoma de Manizales $s$ frecuente pensar la técnica como una simple forma de hacer que, en cierto sentido, resulta independiente de la visión del mundo que se tenga. En tai medida, se suele suponer que el introducir la tecnica en otros contextos culturales diferentes ai Oecidental no necesariamente tendrá consecuencias en su cosmovisión. Sin embargo, ya ha demostrado con suficientes ejemplos la Antropologia que la introđucción de la técnica implica adoptar toda una forma diferente de enfrentarse y valorar la realidad, $y$ que en ocasiones puede incluso tener la consecuencia de arrasar con la cultura aborigen. Al punto que podria afirmarse que el "exportar" la técnica es la forma contemporánea de la "evangelización". Una de las implicaciones de esto es que no resulta tan simple disociar "ser" $y$ "saber", que el saber no supone simplemente un incremento, sino además, 0 principalmente, una modificación en la forma de ser. La pregunta obligada entonces es ¿cuál es esa cosmovisión implicita en la técnica? 
Ha sido sobre todo Heidegger quien en el ámbito contemporáneo ha pensado con mayor agudeza sobre la esencia y naturaleza de la técnica, y una de sus lecciones básicas tiene que ver con la distinción que establece entre lo que fue el concepto de técrica entre los griegos y lo que este concepto ha devenido entre nosotros. Este contraste resulta ineludible a fin de esclarecer o de apuntar en la dirección de la pregunta anterior.

La palabra griega Tejne, de la que deriva la nuestra, técnica, designaba un campo más amplio del que designa entre nosotros, se referia en general al develar $y$ al mismo tiempo resguardar. Era aplicado preferentemente a lo que hoy denominamos "arte", pero incluia no sólo al que producia objetos bellos, poeta o escultor, sino también a quien elaboraba herramientas. Segün Heidegger: "Tejne no sólo es el nombre para el hacer $y$ el saber del obrero manual sino también para el arte, en el sentido elevado, $y$ para las bellas artes, $\mathrm{L}$ a Tejne pertenece al traer-ahi-adelante"', $\mathrm{Y}$ ese retener, resguardar $y$ hacer visibles las "cosas", se consigue a partir del material que proporciona la naturaieza, sin embargo, no implica que para los griegos la naturaleza fuera un simple depósito de material inerte, más bien ha- bria que pensarlo en términos de un cierto dialogo en el que desesperadamente se carece de un lenguaje común, y lo que persigue la tejné es entonces posibilitar la confluencia entre lo natural y lo humano. En otras palabras, la Tejne era entendida como ese saber-hacer desde el cual se apuntaba a una construcción del Hogar humano, a una transformación del mero mundo en Hogar.

Pero desde la Modemidad, $y$ a partir de la Cosmovisión que hace posible el surgimiento de la ciencia, la técnica se ha entendido fundamentaimente como capacidad de dominar. Desde la modernidad, el Universo y la naturaleza dejan de ser vistas como "aigo" con sentido, algo que habra que descifrar y comprender, algo con vida, $y$ se les reduce a un flujo de acontecimientos que tienen lugar segùn leyes inexorables, de modo que conocer se entienda desde el desciframiento de esas leyes, y a partir del supuesto caracter meramente mecánico y funcional de la naturaleza. Lo que persigue ila ciencia moderna es basicamente poder $y$ control sobre la naturaleza. La naturaleza se reduce a un simple objeto útil para satisfacer nuestras necesidades. Heidegget lo piantea de este modo: "Ahora el mundo se aperece como un objeto sobre el que el pensamiento calculador inicia 
sus ataques, ataques que ya nada podrá resistir. La naturaleza se convierte en una única y gigantesca estación de servicio, en fuente de energía para la técnica y la industria modernas. Esta relación fundamentaimente técnica del hombre con el universo surgió primero en el siglo XVII y ello en Europa y sólo en Europa. Y permaneció oculta por largo tiempo a las otras partes del globo. Era totalmente ajena a las anteriores edades y destinos de los pueblos"

Cambia, en consecuencia, el sentido de la técnica y el de su finalidad de traer-ahi-adelante $y$ transformar. Se rompe, digamos asi, el diálogo con la naturaleza, y el papel de la técnica entonces ya se entenderá desde la mediación. El "transforma" se restringe a un sentido mecánico, inmediato, y se entenderá sólo desde la utilidad para el hombre, y ya no desde las "cosas" o en relación con ellas.

Una consecuencia de lo anterior, entre otras, fue el descoyuntamiento, o la separación entre los ámbitos del hacer, del conocer y del actuar. Es decir, la técnica, como ámbito del conocer, se desconectó no sólo del arte sino de la moral. Desde entonces nos parece completamente obvio que el técnico y el cientifico sean éticamente neutrales, $y$ a su vez que lo estético no tenga nada que ver con el conocimiento ni con la utilidad. En términos prácticos esto se ha expresado en un profundo desgarramiento en el hombre contemporáneo, en la imposibilidad de reconciliar el deber y la libertad. El hombre contemporáneo vive fragmentado, escindido entre lo que debe hacer y lo que quiere hacer, entre, por ejemplo, el trabajo y la familia, el conocimiento y el placer, lo bello y lo útil. $\mathrm{Y}$ a pesar de ser conscientes de tal escisión, y del dolor que produce, padecemos cierta incapacidad para reconciliarla.

Lo anterior puede ilustrarse con esta imagen. El cambio que suponen las cosmovisiones que estamos contrastando y que se refleja en el sentido de la palabra técnica, es algo así como que de pronto se comenzara a mirar a la mamá como una seảora que hace un montón de cosas: cocina, plancha, lava, va a misa, se baha, etc; y se dejara entonces de miraria como el centro alrededor del cual se construye eso que se denomina Hogar, como aquella persona que encarna lo Familiar, es decir, ese lugar donde no nos sentimos extraños o extranjeros.

$\mathrm{Y}$ ahora supongan que después de haber descrito a la madre como una mujer que realiza ciertas actividades, recibo una cantidad de sermones de cura donde se me acusa de haber "instrumentalizado" a la madre, entonces decido idealizar ba, le construyo un altar y no dejo que se "ensucie" de rada. Pues bien, esto ejemplifica el otro extremo al que hemos llegado y en el que igual seguimos presos de la incapacidad de reconciliar el deber y la libertad. Piensen en la moda ecologista, en ciertos rasgos suryos, la Tierra convertida en santuario, pero completamente estéril $\mathrm{e}$ inútil para damos abrigo y alimento. $Y$ viene entonces el conflicto entre los defensores a

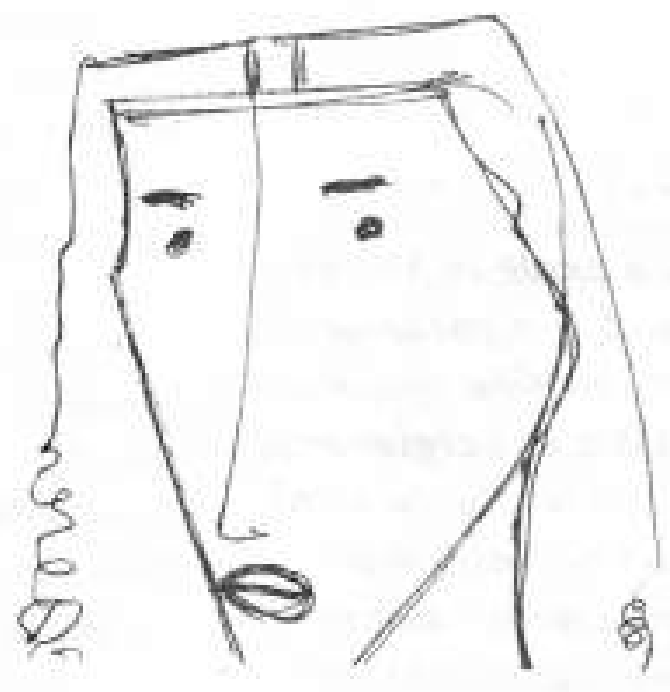


ultranza de la Tierra como santuario, $y$ los que de otro lado ven en cada bosque una fábrica de muebles en potencia.

Para la idea que estamos desarollando, esto significa que el viejo concepto griego de Tejne, fue escindido en la Modernidad en otros dos que, aùn sumados, resultan impotentes en la transformación del mundo en el Hogar humano. Esos dos conceptos son la técnica de un lado y el arte del otro. Al escindirlos, la técnica se vino a ocupar de una pura producción de objetos útiles pero carentes de significación, y el arte se vino a ocupar solamente de la decoración produciendo objetos completamente inútilies. Al final hemos venido a parar en un mundo que antes que Hogar parece más bien o una bodega o un museo. En ambos casos se trata de objetos carentes de vida, de significación. Para Gadamer, "vivimos en el moderno mundo industrial. $Y$ este mundo no sólo ha expulsado al margen de nuestra existencia las formas visibles del rito y del culto; también ha destruido, ademas, lo que una cosa es. [...] ya no hay cosas con las que tratemos. Todo lo que hay son articulos que se pueden comprar tantas veces como se quiera, porque se los puede producir tantas veces como se quiera, hasta que deje de fabricarse el mo- delo. Asi son la producción y el consumo modernos. Lo cierto es que estas cosas ya sólo se fabrican en serie, que sólo se las vende por medio de anuncios lanzados a lo grande y que, cuando se rompen, se las tira. Peroen elias no hacemos la experiencia de lo que es una cosa. No hay ya nada en ellas que se haya convertido en presencia, que se sustraiga a la sustinubilidad, ninguin fragmento de vida, ninguna parte historica") . Incluso, algunos articulos que baftados por la pátina del
El hombre contemporáneo vive fragmentado, escindido entre lo que debe hacer y lo que quiere hacer, entre, por ejemplo, el trabajo $y$ la familia, el conocimiento y el placer, lo bello y lo útil. tiempo habian logrado cierta vida y capacidad de evocación, han terminado meramente en objetos inemes gracias a la "idealización" del pasado, reflejada en la moda de anticuarios, en la valoración que se hace de lo antiguo sólo porque es antiguo.

En el àmbito del saber esto también vino a implicar una ruptura. De un modo que podria parecer metaförico, pero que no lo es, Foucault afima en Las Palabras $y$ las cosas, que el hombre es una creación reciente, surgida sólo a partir de una cierta manera de ordenar la realidad, pero que una vez accedamos a otro ordenamiento, desaparecera. Esto no significa que a nosotros nos hayan inventado en el siglo pasado y que en unos años toda la humanidad desaparecera. Lo que Foucault quicre afimar es que eso 
que denominamos Hombre, lo que hoy entendemos por esa palabra, es resultado del mismo desgarramiento que con referencia a la técnica hemos aludido. Del mismo modo que prácticamente se oponen los sentido de la palabra técnica entre los antiguos y nosotros, igual ocurre con el concepto de Hombre.

Ya señalaba antes, con el ejemplo que ponia de la madre, que hay dos formas de entenderla, una, la madre como aquella mujer donde son indisociables el amor y el hacer, aquella mujer que, como dice una canción de Facundo Cabral, "nos dice te quiero con la camisa planchada"; $y$ otra forma de entenderla es idealizarla, es desligar el amor $y$ el hacer $y$ definirla ya sea por uno o por otro de los términos, o como puro amor, o como puro hacer. Pues bien, algo similar ocurre con el concepto de Hombre, a lo que denominamos Hombre y que ha devenido en objeto de estudio de las Ciencias Humanas y Sociales, es el resultado de una "idealizaciön", valga decir, escisión. Es en este sentido que el Hombre es una invención reciente: definido desde una abstracción que lo desliga de manera absoluta de la naturaleza, y que lo obliga a elegir entre ser completamente libre, o una máquina. De un mundo lleno, en donde el hombre, siendo uno más entre todo lo existente estaba en diálogo con todo, hemos llegado a una completa soledad, a definimos desde y por el aislamiento, el diálogo ha devenido en monólogo (con ello, dicho sea de paso, tambièn la palabra vino a entenderse de una manera instrumental, ya no como puente ni comunicación). Surge entonces un nuevo objeto del saber: algo que no es simple materia inerte, algo completamente distinto a la enorme maquina que es la naturaleza, algo vivo, que ama, desea, sueña y se apasiona. $Y$ se abre asi entonces la posibilidad y la discusion acerca de dos tipos de ciencia, una que se ocupa de los objetos inertes que, mecanica e inexorablemente obedecen a leyes universales y necesarias, y otra que se ocupa del hombre, es decir, de lo singular, de lo que posee autonomia y voluntad, y en consecuencia es libre. De modo que ia misma idealización que construyó al hombre corno objeto de un saber, abrió la posibilidad de operar sobre él, la misma mirada funcionalista que abrió el camino a las ciencias naturales. Desde el siglo pasado se discute si es válido denominar ciencia a un cierto saber que no es medible, con lo que muchos han considerado entonces que elevar ese saber efoctivamente a ciencia depende de hacer cuantificable aquello de lo que se ocupan las ciencias humanas. Para Rorty: "La forma habitual de tratar la relación entre ciencias humanas y ciencias naturales es sugerir que se dividan la cuitura entre si - las ciencias naturales se ocuparian de la parte cognitiva seria e importante (aquella en que cumplimos nuestras obligaciones con la racionalidad) y las ciencias humanas de todo lo demás. La idea que está detrás de esta división es que el conocimiento en sentido estricto debe tener un Logos y que un Logos sólo puede ser dado por el descubrimiento de un método de conmensuración. La idea de conmensurabilidad está incluida en la noción de cognición auténtica, lo que es sólo cuestión de gusto o de opinión no tiene por qué caer necesariamente dentro de la responsabilidad de las ciencias naturales, y por el contrario, lo que las ciencias naturales no pueden hacer conmensurable se desprocia como meramente subjetivo" 
Desde la Modernidad hernos pensado al Hombre como un sujeto encerrado en si mismo, para quien su "humanidad" es un dato. es algo dado que no se afecta ni se expresa ni en su hacer ni en su saber. Como si se partiera del hecho de que nacemos siendo humanos y que por lo tanto la educación juega sólo un papel secundario, correctivo pero no formador en el sentido profundo de la palabra. Se piensa pues la educación como un agregar o sumar a lo que ya somos, una cierta habilidad en el hacer, $y$ un cierto saber. $Y$ cuando en ocasiones. se le abre un espacio a la "formación", resulta estéril, porque a fin de cuentas lo que se hace no es más que reforzar la escisión; la ideologia que parte del ser humano como un ser escindido queda inconmovible.

En los últimos años, sin cmbargo, y desde diferentes ámbitos, incluidas las ciencias naturales, ha venido ganado fuerza la idea de pensar al Hombre y al Mundo de una manera integral, aun al riesgo de asumir su enorme complejidad como algo ineludible. Al punto que podria afirmarse sin ser atrevido, que quienes aun no admiten la cuestionabilidad de los limites entre, por ejemplo, ciencias naturales y humanas, razón y sensibilidad, naturaleza y cultura, objetividad y subjetividad, ser y saber, siguen presos de una visión anacrónica, la de la modernidad.
Hoy, luego del desenmascaramiento del trasfondo ideológjcoy político de la ciencia llevado a cabo, sobre todo, por la Escuela de Frankfurt (Adorno, Marcuse, Habermas), luego del desencanto frente a lo prometido desde la llustración por la ciencia, de que éste seria un mundo más justo y que seriamos más felices, luego de que la propia ciencia natural llegara al convencimiento de que no puede seguir marginando lo singular y lo complejo, prácticamerte ya no tiene sentido insistir en definir al hombre desde la escisión y restringirlo a la idealización.

También al interior de las propias ciencias naturales se han venido volviendo problemáticos los limites y la definicion de objeto y su-
También al interior de las propias ciencias naturales se han venido volviendo problemáticos los limites y la definición de objeto y sujeto...

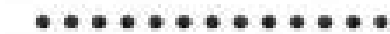
jeto, $\mathrm{y}$ cada vez más las ciencias han venido ocupándose de lo singular. El fisico norteamericano Fritoj Capra incluso se atreve a afirmar que estamos en las fronteras de un nuevo paradigma cientifico, que la mirada galileana esta siendo desplazada por la que denomina una visión ecologista del saber. En defensa de su tesis menciona los avances en Teoria de Sistemas, en Matemática de la complejidad y el inusitado interés que ha vuelto a cobrar el enfoque morfológico en Biologia. Se trata, dice, de una superación del ideal reduccionista de la ciencia, en favor de un planteamiento holista. En una dirección similar, cada vez cobra más fuerza la pro- 
puesta de Edqar Morin acerca del pensamiento compiejo. Para Morin: "Hay un nueva ignorancia ligada al desarrollo mismo de la ciencia, hay una nueva ceguera ligada ail uso degradado de la razón i y estos) sienen un carácter comùn que resulta de un modo mutilante de organizacion del conocimiento, incapaz de reconocer y de aprehender la complejidad de lo real"s.

Segun Morin, carece de sentido que sigamos pensando al hombre desarticulado de la esfera biologica y fisica, es necesario integrar fisica, biologia y socioantropologia. Y se pregunta: "¿Podemos quedar satisfechos al no concebir al individuo más que excluyendo la sociedad, a la sociedad excluyendo la especie, a lo humano excluyendo la vida, a la vida exchuyendo la physis y a la fisica excluyendo la vida? ¿Se puede aceptar que los procesos locales en precision vayan acompañados de un halo de imprecision de las formas globales y las articulaciones? ¿Se puede aceptar que la medida, la previsión, la manipulacion hagan retroceder la inteligibilidad? iSe puede aceptar que las cuestiones clave sean enviadas a las mazmorras? ¿Se puede aceptar que el conocimiento se funde en la exclusion del cognoscente, que el pensamiento se funde en la exclusion dei pensante, que el sujeto sea excluido de la construccion del objeto? QQue la ciencia sea totalmente inconsciente de su insercion y de su determinacion sociales? *

Si bien el problema en la Modernidad, y al que se asocia el nacimiento de ia ciencia, es el dominio y control sobre ia naturaleza, hoy ese problema esta en buena medida resueitoy lo que urge es controlar y dominar la técnica. Y esto implica replegarnos sobre nosotros mismos y formular con un sentido renovado la vieja pregunta ¿qué es el hombre?

Sólo desde esta pregunta será posible alcanzar que la ciencia y la técnica estén al servicio del hombre y no a la inversa. Sólo desde esta pregunta sera posible volver a conciliar el deber y el deseo, y asumir la tarea de producción como una simultanea transformación y construcción del Hogar hummo. Pero es necesario aclarar que esta critica a la modernidad desde el contraste con el sentido griego de tejné, no debe entenderse como si se estuviera proponiendo como solucion un regreso imposibte al pasado. La ciencia y la técnica forman ya parte ineludible de nuestro mundo, y asi mismo hay que admitir que desde los ideales humanistas de la itustración, hemos ganado, por ejemplo, un sentido mas amplio de la libertad. El constraste pues, no tiene ta intención de ponemos a elegir entre ia modernidad y la antigüedad ciásica, más bien se trata es de reconciliarlas. Asi como tampoco se trata de elegir entre ética y ciencia.

Por supuesto la pregunta que queda, el reto, es ¿cómo? ¿Qué papel juega la educacion, y en concreto la Universidad, en ese redimensionamiento de la técnica y en la recuperación de la pregunta por el Hombre? ¿Basta con, por ejemplo, aumentar las asignaturas dedicadas a las Humanidades? ¿Y, dado el caso que la Universidad diera con un excelente modelo capaz de lograr la integración, no se veria desperdiciado su esfuerzo mientras siguiera inmodificable el contexto social? Pero, de otro tado, ¿cumo se modifica el contexto social sino es a partir del riesgo de algunos de los que lo conforman?

' HEIDEGGER, Martin. Articulos y conferencius (Nio 5). Barcelona : Odós, 1994. p. Is

I HEIDEGGER Martin Serenidad. En: Revista Colombirna de psicologia. Universidad Nacional de Colombia, Bogota. \# 3, aho MCMDCIV.

3 GADAMER, Hans-Georg Estética y hermeneutica. (Colección Metrópolis). Madrid : Técnos, 1996. p. 91.

- RORTY, Rorty. La filosofia y et espejo de ia naturaien. Mudrid : Castedint, 1995. p. 291

" MORIN, Edgar, Introducción al pensamiento compiejo. Barcelona : Gedisa, 1996. p. 28

- MORIN. Edagar. El Metodo: la naturaieza de la naturo leza. 4a edición. Mndrid: Cátedra. 1997, t. L p. 27. 